\title{
Improving dynamic tomography, through Maximum a Posteriori estimation
}

\author{
Glenn R. Myers*a, Matthew Geleta ${ }^{b}$, Andrew M. Kingston ${ }^{a}$, Benoit Recur ${ }^{a}$ and Adrian P. \\ Sheppard ${ }^{a}$ \\ ${ }^{a}$ The Australian National University, Canberra ACT 0200, Australia; \\ $b$ The University of Melbourne, 1-100 Grattan Street, Parkville VIC 3010, Australia
}

\begin{abstract}
Direct study of pore-scale fluid displacements, and other dynamic (i.e. time-dependent) processes is not feasible with conventional X-ray micro computed tomography $(\mu \mathrm{CT})$. We have previously verified that a priori knowledge of the underlying physics can be used to conduct high-resolution, time-resolved imaging of continuous, complex processes, at existing X-ray $\mu \mathrm{CT}$ facilities. In this paper we present a maximum a posteriori (MAP) model of the dynamic tomography problem, which allows us to easily adapt and generalise our previous dynamic $\mu \mathrm{CT}$ approach to systems with more complex underlying physics.
\end{abstract}

Keywords: Computed tomography, dynamic tomography, fluid flow, 4D tomography, maximum a posteriori, Bayesian, micro computed tomography, time-resolved tomography

\section{INTRODUCTION}

$\mathrm{X}$-ray micro-computed tomography $(\mu \mathrm{CT})$ is a powerful tool for the non-destructive study of internal structures at the micron-scale. ${ }^{1}$ In many cases we are interested not only in the internal structure of a sample, but also in its dynamic (i.e. time-dependent) evolution. In this paper we focus on multiphase displacements of immiscible fluids, in microporous media. These displacements are central to oil production since the manner in which water displaces oil determines the fraction of oil that can be recovered. ${ }^{2}$ Similarly, the micron-scale fluid physics of the rhizosphere (the soil and water immediately surrounding a plant root) has implications for surface runoff, soil compaction and erosion, and water/nutrient uptake by plants.

Unfortunately, conventional X-ray $\mu \mathrm{CT}$ techniques cannot be used to study dynamic processes such as multiphase fluid flow. In X-ray $\mu \mathrm{CT}$ an object is illuminated with penetrating radiation, and we collect radiographs at a number of different viewing angles. From these radiographs we can use a conventional reconstruction algorithm* to reconstruct the 3D distribution of the X-ray linear attenuation coefficient. ${ }^{1,3,4}$ If the sample changes during data acquisition then the radiographs will be inconsistent with one another, causing blurring in the reconstruction. The time required to collect a full set of radiographs (i.e. the time the sample must remain static) scales with the desired spatial resolution of the reconstruction, ${ }^{1}$ effectively preventing imaging with a high spatial and temporal resolution.

The behaviour of complex systems is constrained a priori, by the physics underlying the system. Previous work has demonstrated that this a priori knowledge can be used to resolve inconsistencies in the measured data, improving temporal resolution by an order of magnitude..$^{5-9}$ We have previously presented and verified an "dynamic tomography" algorithm for the 4D imaging of continuous two-phase fluid displacement in complex 3D materials. ${ }^{7,10,11}$ Dynamic tomography generates a time-series of volume images: a 4D "movie" where every frame is a full 3D volume rendering. This algorithm makes use of an a priori model of the underlying physics, ${ }^{7}$ and has been applied to the study of water drainage and flow hysteresis in Bentheimer sandstone. These results were subsequently verified using ground-truth experiments conducted at the $\mathrm{ANU} \mu \mathrm{CT}$ facility. ${ }^{11}$ Unfortunately,

\footnotetext{
* E-mail: glenn.myers@anu.edu.au

${ }^{*}$ Examples include: Filtered backprojection (FBP), algebraic reconstruction technique (ART), simultaneous iterative reconstruction technique (SIRT), simultaneous algebraic reconstruction technique (SART), or Maximum likelyhood (ML) inversion.
}

Developments in X-Ray Tomography IX, edited by Stuart R. Stock, Proc. of SPIE Vol. 9212, 921211 - (c) 2014 SPIE · CCC code: 0277-786X/14/\$18 - doi: 10.1117/12.2061604 
this dynamic tomography algorithm is empirically derived. Consequently, it is difficult to adapt or generalise the algorithm to study more complex dynamic processes such as multi-phase flow, or water uptake by plant roots.

In the context of an imaging problem such as $\mu \mathrm{CT}$ reconstruction, Bayesian statistics can be used to derive an objective function that can be maximised to find the most probable solution, given the measured data and any $a$ priori knowledge. ${ }^{4}$ This mathematically rigorous process is known as maximum a posteriori (MAP) estimation: it requires an accurate description of the imaging system (in the form of a conditional probability distribution relating sample properties to observable measurements), and a prior probability distribution capturing any a priori information. In the absence of a priori information, MAP estimation becomes maximum likelihood estimation.

Previous Bayesian approaches to CT either: do not allow the sample to change continuously and nonperiodically; ${ }^{4}$ or assume that changing structures rarely overlap in the measured radiographs (see digital subtraction angiography techniques, such as HYPR, HYPRIT, I-HYPR, 4D DSA, etc).$^{8,9}$ Neither approximation is suitable for our purposes. In this paper we present a new framework for dynamic tomography of fluid flows in microporous materials. We derive an expectation-maximisation algorithm for MAP estimation (MAP-EM) that simultaneously reconstructs the dynamic evolution of the sample, and segments the reconstruction by material composition. As in our previous work, we begin by subtracting and removing the static component of the sample.

We demonstrate that when applied to the study of two-phase flow, this MAP-EM algorithm reduces to a form much like our previous, empirically-derived method. Thus, the work in this paper provides a sound mathematical basis for our prior dynamic tomography work. Furthermore, the MAP-EM approach is more general than our previous efforts: we discuss how the MAP-EM algorithm can be applied to more complex physical systems. We perform ground-truth reconstructions that verify the results of our new MAP-EM algorithm, using experimental $\mu \mathrm{CT}$ data. We compare the performance of MAP-EM to our previous dynamic tomography algorithm, and conventional $\mu \mathrm{CT}$ reconstruction techniques. As before, use of a priori information significantly improves temporal resolution relative to conventional $\mu \mathrm{CT}$ reconstruction techniques. Finally, we test a variant of our MAP-EM algorithm that is suitable for the study of multiphase flow.

\section{IMAGING FLOW IN X-RAY $\mu$ CT}

We consider a sample placed on the rotation stage of a typical lab-based, fine-focus X-ray $\mu \mathrm{CT}$ imaging system. We describe the linear X-ray attenuation coefficient of this sample as the sum of both static $\mu_{s}(\mathbf{x})$ and dynamic $\mu(\mathbf{x}, t)$ components, where $\mathbf{x}=\left(x_{1}, x_{2}, x_{3}\right)$ are 3D Cartesian coordinates and $t$ is time. As the sample begins to change (e.g. a drainage pump is switched on), it is illuminated with X-rays. The sample attenuates these X-rays, and on the far side of the sample a $2 \mathrm{D}$ position-sensitive X-ray detector records a radiograph. The sample stage rotates through a number of viewing positions $\theta$, and we collect a time-sequence of radiographs at times $t \in T_{i}$. In a helical (or other non-circular) scanning geometry, the stage will also translate vertically during acquisition. ${ }^{12}$

When the experiment is complete we allow the sample to reach a static, equilibrium state. For example, it may be fully drained of fluid, or the pump may be switched off. We then image this state using standard $\mu \mathrm{CT}$ methods, producing an estimate of $\mu_{s}(\mathbf{x})$.

Finally, we pre-process our time-sequence of radiographs to correct for mis-alignments, source drift, clear and dark field signals, etc. ${ }^{13-15}$ We also simulate the signal due to the known static component $\mu_{s}(\mathbf{x})$, and remove it from these radiographs. ${ }^{7}$ The resulting intensity images $I\left(\mathbf{r}, \theta, t \in T_{i}\right)$ contain only signal due to the dynamic component of the sample. Here, $\mathbf{r}=\left(r_{1}, r_{2}\right)$ are 2D Cartesian coordinates in the plane of the detector.

\section{MAXIMUM A POSTERIORI RECONSTRUCTION}

Given measured data $I\left(\mathbf{r}, \theta, t \in T_{i}\right)$ and $\mu_{s}(\mathbf{x})$, we desire to reconstruct the $4 \mathrm{D}$ dynamic component of the sample $\mu(\mathbf{x}, t)$, at a series of instants $t \in T_{\mu}$. We expect this problem to be severely ill-posed, due to the constantlychanging nature of the sample. Accordingly, we will make use of a priori, physics-based constraints on the dynamic evolution of $\mu(\mathbf{x}, t)$, and attempt to find the maximum a posteriori estimate

$$
\hat{\mu}\left(\mathbf{x}, t \in T_{\mu}\right)=\underset{\mu}{\arg \max } P\left(\mu \mid I, \mu_{s}\right),
$$


i.e. we search for the reconstruction that is most probable, given the measured data. In order to solve this problem we introduce two new latent (i.e. unobserved) variables, and work within an expectation-maximisation framework.

The first latent variable is the composition $m\left(\mathbf{x}, t \in T_{\mu}\right) \in D$, which is the material present in the sample at position $\mathbf{x}$ and time $t$. These materials are chosen from some dictionary $D$. This latent variable allows us to introduce a priori information about the composition of a sample, and is usually a quantity of interest in-and-of itself. For example, in a drainage experiment we are interested in fluid distribution more than linear attenuation coefficient.

We are attempting to reconstruct the dynamic component of our sample at times $t \in T_{\mu}$, from radiographs taken at times $t \in T_{i}$. Due to data storage constraints, we are likely to choose far fewer times $t \in T_{\mu}$ than we have radiographs. This means that we require some way of inferring a radiograph taken at time $t \notin T_{\mu}$, from our reconstruction $\mu\left(\mathbf{x}, t \in T_{\mu}\right)$. To do so, we introduce second latent variable: a similarity measure $\tau\left(t_{0} \in T_{i}, t_{1} \in T_{\mu}\right)$, such that $P\left[\tau\left(t_{0} \in T_{i}, t_{1} \in T_{\mu}\right) \mid I, \mu\right]$ quantifies our confidence that a radiograph at time $t_{0}$ can be reconstructed from a volume at time $t_{1}$.

Given these latent variables, we can formulate a maximum a posteriori expectation-maximisation (MAP-EM) algorithm, which iteratively converges to $\hat{\mu}\left(\mathbf{x}, t \in T_{\mu}\right)$. At iteration $(n)$, we calculate and then maximise the expectation function

$$
\begin{aligned}
f^{(n)}\left(\mu \mid \mu^{(n)}, I, \mu_{s}\right) & =\sum_{\tau} \sum_{m} P\left(\tau, m \mid \mu^{(n)}, I, \mu_{s}\right) \ln \left[P\left(\tau, m, I, \mu_{s} \mid \mu\right)\right] . \\
\mu^{(n+1)} & =\underset{\mu}{\arg \max }\left[f^{(n)}\left(\mu \mid \mu^{(n)}, I, \mu_{s}\right)+\ln [P(\mu)]\right] .
\end{aligned}
$$

We note that: (i) in X-ray CT, composition is inferred from the linear attenuation coefficient, so if $\mu_{s}$ and $\mu$ are known then $m$ is independent of $I$ and $\tau$; and (ii) similarly, if $\mu$ is known then $P(I, \tau)$ is independent of $\mu_{s}$ and $m$. We can then use Bayes theorem to derive the following iterative MAP-EM algorithm:

$$
\begin{aligned}
\mu^{(n+1)}=\underset{\mu}{\arg \max } & \sum_{\tau} P\left(\tau \mid I, \mu^{(n)}\right) \ln [P(I \mid \tau, \mu)] \\
& +\sum_{\tau} P\left(\tau \mid I, \mu^{(n)}\right) \ln [P(\tau \mid \mu)] \\
& +\sum_{m} P\left(m \mid \mu_{s}, \mu^{(n)}\right) \ln \left[P\left(\mu_{s}, m \mid \mu\right)\right] \\
& +\ln [P(\mu)] .
\end{aligned}
$$

We will discuss each term in this algorithm individually, presenting a physical interpretation and examples of how it may be calculated in practice.

\subsection{Data consistency}

The first term in Eqn. (1) attempts to maximise consistency between the pre-processed measured radiographs $I\left(\mathbf{r}, \theta, t \in T_{i}\right)$, and the reconstruction $\mu\left(\mathbf{x}, t \in T_{\mu}\right)$. In order to calculate this term we require: (i) some means of estimating $\tau$; and (ii) a noise model for our imaging system.

The drainage experiment presented later in this paper is dominated by Haines jumps: a sequence of rapid and discrete flow events. ${ }^{16-20}$ The jump time is approximately proportional to the mass of the fluid displaced in that jump, ${ }^{20}$ and tends to be on the order of milliseconds. The time between jumps is typically larger, on the order of a few seconds. ${ }^{17,20}$ This behaviour occurs in fluids such as water, oil, and paraffin, and is viscosity and density dependent. ${ }^{19}$

In cases like these, where the dynamic process is dominated by quantised, temporally localised movements, these movements make appropriate markers for temporal partitioning. Haines jumps can be found by tracking changes in the total recorded attenuation $\left\|I\left(\mathbf{r}, \theta, t \in T_{i}\right)\right\|_{L_{1}}$ of the sample, as long as the detector's field of view does not change too rapidly between radiographs. Having partitioned our timeline according to these jumps, we 
use a similarity measure that reduces with temporal separation, and vanishes entirely across partition boundaries (i.e. Haines jumps):

$$
\begin{aligned}
P\left[\tau\left(t_{0} \in T_{i}, t_{1} \in T_{\mu}\right) \mid I, \mu^{(n)}\right] & =\Gamma\left(t_{0}, t_{1}\right), \\
1-\Gamma\left(t_{0}, t_{1}\right) & \propto\left|t_{0}-t_{1}\right|, \text { if } t_{0} \text { and } t_{1} \text { are not separated by a Haines jump, } \\
\Gamma\left(t_{0}, t_{1}\right) & =0, \text { otherwise. }
\end{aligned}
$$

To calculate $P(I \mid \tau, \mu)$, we make the X-ray projection approximation. This models the X-ray/sample interactions using a set of line integrals along the ray paths, ignoring diffraction and other wave-like behaviours within the object. For the sake of simplicity we further assume that noise in the projected attenuation follows a Gaussian distribution with standard deviation $\sigma_{1}$, i.e. that:

$$
P\left[I\left(\mathbf{r}, \theta, t_{0} \in T_{i}\right) \mid \tau\left(t_{0}, t_{1} \in T_{\mu}\right), \mu\left(\mathbf{x}, t \in T_{\mu}\right)\right] \propto \exp \left\{-\frac{\left[\ln I\left(\mathbf{r}, \theta, t_{0}\right)-\left(\mathcal{P}_{\theta} \mu\right)\left(\mathbf{r}, t_{1}\right)\right]^{2}}{2 \sigma_{1}{ }^{2}}\right\},
$$

where $\mathcal{P}_{\theta}$ is the X-ray projection operator for a cone-beam fine-focus source, at viewing position $\theta$. Parallelbeam X-ray sources (e.g. synchrotrons) may be modelled as a limiting case of this operator. ${ }^{3}$ Generalisations of Eqn. (3) may use more physically accurate noise models (e.g. Poisson noise in recorded intensity), or may include additional physics (e.g. free-space X-ray refraction between object and detector).

Given Eqs. (2) and (3), we can calculate the first term in Eqn. (1). Ignoring for the moment the other three terms, we may take an iterative gradient-descent (or in this case, gradient ascent) approach to finding $\mu^{(n+1)}$ :

$$
\mu^{(l+1)}\left(\mathbf{x}, t_{1} \in T_{\mu}\right)=\mu^{(l)}\left(\mathbf{x}, t_{1}\right)+\sum_{t_{0} \in T_{i}} \Gamma\left(t_{0}, t_{1}\right) \mathcal{B}\left\{\ln \left[I\left(\mathbf{r}, \theta, t_{0}\right)\right]-\left(\mathcal{P}_{\theta} \mu^{(l)}\right)\left(\mathbf{r}, t_{1}\right)\right\},
$$

where $\mathcal{B}$ is the backprojection operator, and the superscript $(l)$ denotes the $l$ th iteration of gradient descent to find $\mu^{(n+1)}$. This iterative update is a SART-like method, in which the similarity measures $\Gamma\left(t_{0}, t_{1}\right)$ take on the role of interpolation weights.

\subsection{Temporal re-partitioning}

If the times $T_{\mu}$ at which we attempt to reconstruct the object are fixed a priori, then $P(\tau \mid \mu)$ is flat and the second term in Eqn. (1) vanishes for our choice of $P\left[\tau\left(t_{0} \in T_{i}, t_{1} \in T_{\mu}\right) \mid I, \mu^{(n)}\right]$ above. Future work will explore this term as a means of adaptively selecting reconstruction times $T_{\mu}$ : this is potentially useful for cases where the characteristic nature of the motion (e.g. Haines jumps) is not understood in advance.

\subsection{Material classification and compositional priors}

The third term in Eqn. (1) allows us to incorporate information from the static component of the sample, as well as any compositional a priori information.

In a two-phase fluid drainage experiment, our materials dictionary contains three entries $D=\{$ rock, fluid, air . The rock scaffold is un-changed and impermeable throughout the drainage process, and we expect to be able to find its support $\Omega$ from the static CT data $\mu_{s}(\mathbf{x})$. If the chemical composition of the fluid is known in advance, then we can calculate an estimate of its refractive index $\mu_{\text {fluid }}$. Assuming a gaussian noise distribution with standard deviation $\sigma_{2}$ in our reconstructed X-ray attenuation coefficient, we thus have:

$$
\begin{aligned}
\sum_{m} P\left[m \mid \mu_{s}, \mu^{(n)}\right] \ln \left[P\left(\mu_{s}, m, \mu\right)\right] & \propto-\mu\left(\mathbf{x}, t \in T_{\mu}\right)^{2}, \text { if } \mathbf{x} \in \Omega, \\
& \propto-\mu\left(\mathbf{x}, t \in T_{\mu}\right)^{2} \exp \left\{-\frac{\mu^{(n)}\left(\mathbf{x}, t \in T_{\mu}\right)^{2}}{2 \sigma_{2}{ }^{2}}\right\} \\
& -\left[\mu\left(\mathbf{x}, t \in T_{\mu}\right)-\mu_{\text {fluid }}\right]^{2} \exp \left\{-\frac{\left[\mu^{(n)}\left(\mathbf{x}, t \in T_{\mu}\right)-\mu_{\text {fluid }}\right]^{2}}{2 \sigma_{2}{ }^{2}}\right\}, \text { otherwise. }
\end{aligned}
$$

This formulation privileges the static information $\mu_{s}$ and $\Omega$ over the present guess at the dynamic reconstruction $\mu^{(n)}$, on the grounds that the static data can be measured over a longer period of time, with a higher resolution and signal-to-noise ratio than the dynamic measurements $I\left(\mathbf{r}, \theta, t \in T_{i}\right)$. 


\subsection{Regularisation}

The final term in Eqn. (1) is a regularisation parameter, representing a priori information about the dynamic component of the sample. In the case of our drainage experiment below, we expect the fluid to take up a small fraction of the overall volume. This sparsity constraint (c.f. compressed sensing ${ }^{21}$ ) can be encoded using the Laplace density function

$$
P\left[\mu\left(\mathbf{x}, t \in T_{\mu}\right)\right] \propto \exp \left[-\frac{\left|\mu\left(\mathbf{x}, t \in T_{\mu}\right)\right|}{a}\right],
$$

where $a$ is a scaling parameter.

\subsection{A reconstruction algorithm for two-phase flow}

Having examined each term in Eqn. (1) in the context of a two-phase flow experiment, we are in a position to present a dynamic CT MAP-EM reconstruction algorithm for two-phase flow in micro-porous materials:

1. From the static CT data $\mu_{s}(\mathbf{x})$, determine the support of the rock scaffold $\Omega$.

2. From the dynamic radiographs $I\left(\mathbf{r}, \theta, t_{0} \in T_{i}\right)$, locate large Haines jumps to determine the interpolation coefficients $\Gamma\left(t_{0}, t_{1}\right)$.

3. Iteratively solve Eqn. (1) using a gradient descent loop (iteration $l$ ) nested within an expectation maximisation loop (iteration $n$ ):

(a) A gradient descent step (with tunable step sizes $\alpha_{j}$ ) to fit the measured data and compositional priors

$$
\begin{aligned}
\mu^{(n: l+0.5)}\left(\mathbf{x}, t_{1} \in T_{\mu}\right) & =\mu^{(n: l)}\left(\mathbf{x}, t_{1}\right)+\alpha_{1} \sum_{t_{0} \in T_{i}} \Gamma\left(t_{0}, t_{1}\right) \mathcal{B}\left\{\ln \left[I\left(\mathbf{r}, \theta, t_{0}\right)\right]-\left(\mathcal{P}_{\theta} \mu^{(n: l)}\right)\left(\mathbf{r}, t_{1}\right)\right\} \\
& -\alpha_{2} \mu^{(n: l)}\left(\mathbf{x} \in \Omega, t_{1}\right)-\alpha_{3} \mu^{(n: l)}\left(\mathbf{x} \notin \Omega, t_{1}\right) \exp \left\{-\frac{\mu^{(n)}\left(\mathbf{x} \notin \Omega, t_{1}\right)^{2}}{2 \sigma_{2}{ }^{2}}\right\} \\
& -\alpha_{3}\left[\mu^{(n: l)}\left(\mathbf{x} \notin \Omega, t_{1}\right)-\mu_{\text {fluid }}\right] \exp \left\{-\frac{\left[\mu^{(n)}\left(\mathbf{x} \notin \Omega, t_{1}\right)-\mu_{\text {fluid }}\right]^{2}}{2 \sigma_{2}{ }^{2}}\right\} .
\end{aligned}
$$

(b) A compressed sensing step to satisfy our a priori sparsity constraint, using the soft-thresholding operator $\mathcal{T}$

$$
\mu^{(n: l+1)}\left(\mathbf{x}, t_{1} \in T_{\mu}\right)=\left[\mathcal{T} \mu^{(n: l+0.5)}\right]\left(\mathbf{x}, t_{1}\right) .
$$

(c) If the gradient descent loop has converged, update the outer loop

$$
\mu^{(n+1: l=0)}\left(\mathbf{x}, t_{1} \in T_{\mu}\right)=\mu^{(n: l \rightarrow \infty)}\left(\mathbf{x}, t_{1} \in T_{\mu}\right)
$$

This result demonstrates that when the MAP-EM formulation in Eqn. (1) is applied to two-phase flow in micro-porous materials, it produces an algorithm similar to our previous, empirically-derived result in Ref. 7. Unlike our previous efforts this MAP-EM algorithm has a clear derivation, and thus permits generalisation beyond the domain of two-phase flow. This algorithm represents a "limiting case" of the MAP-EM formulation in Eqn. (1): further work will explore how alterations to the prior probability distributions (e.g. allowing $T_{\mu}$ to vary as the algorithm converges, or expanding the materials dictionary) allow imaging of more complex physical systems. 

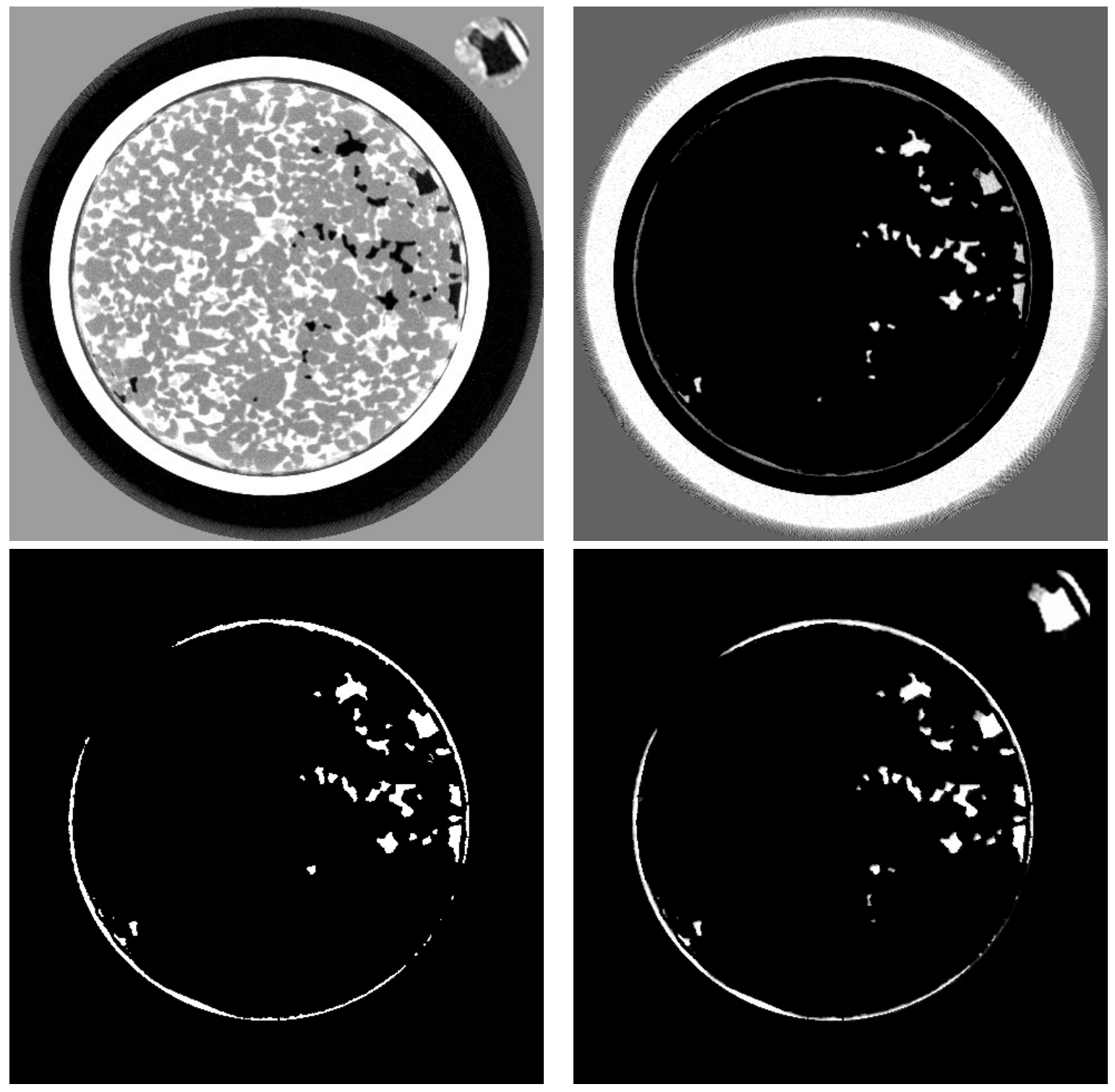

Figure 1. 2D (x-y) slices through the $5 \mathrm{~mm}$ diameter Bentheimer plug. Top left: FBP ground-truth reconstruction showing void (black), fluid (white) and rock/quartz (grey). Inset top-right of image shows a pore which is partially filled with impermeable scaffold. Top right: FBP ground-truth reconstruction segmented to show void (white). Bottom left: Reconstruction using algorithm from Ref. 7. Bottom right: MAP-EM reconstruction showing void (white), using every tenth radiograph. Inset shows successful reconstruction of the "partially empty" voxels where pores were partially filled with impermeable scaffold. 

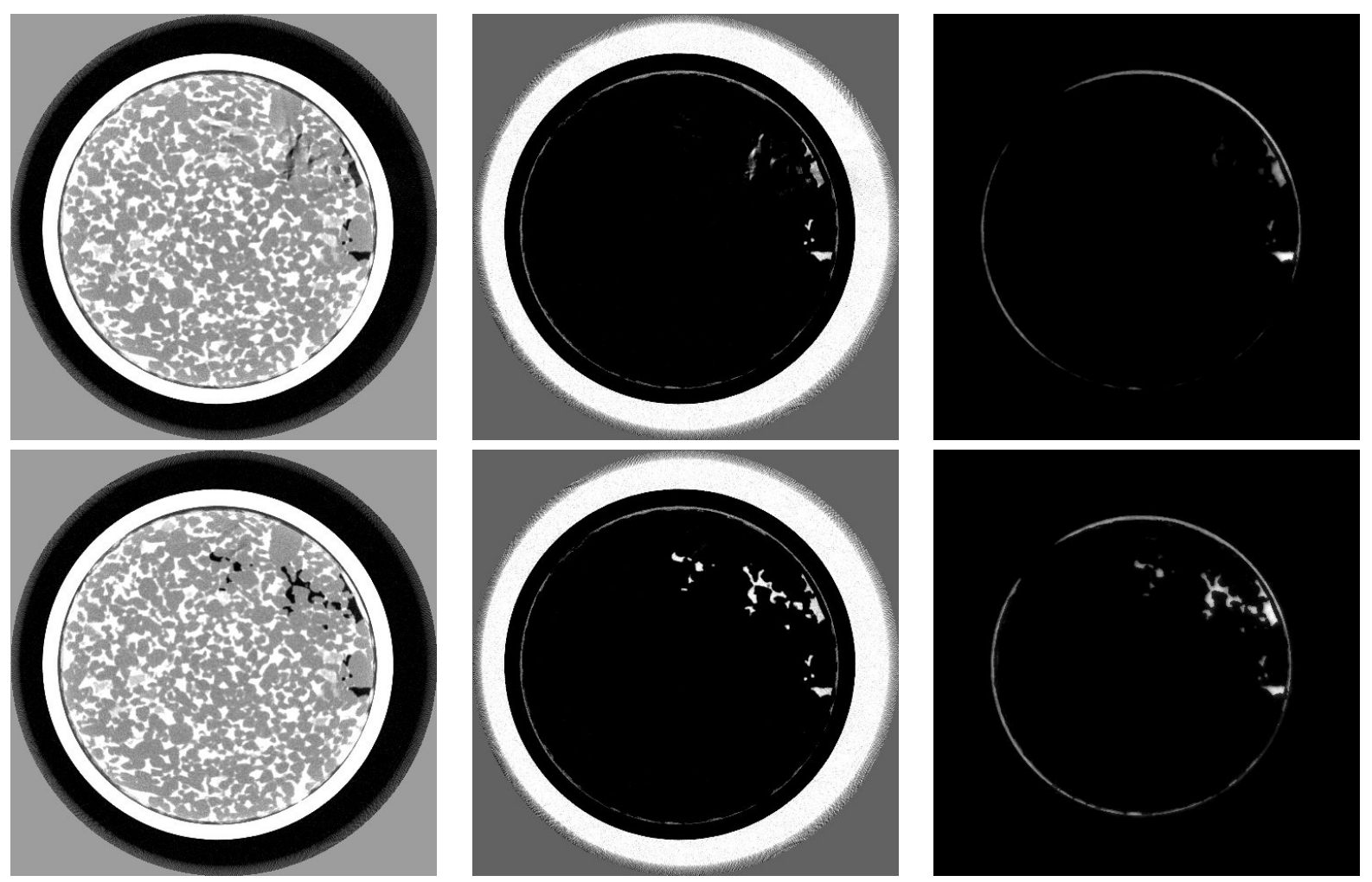

Figure 2. 2D ( $\mathrm{x}-\mathrm{y})$ slices through the $5 \mathrm{~mm}$ diameter Bentheimer plug, at two consecutive time steps (top to bottom). Left: FBP ground-truth reconstruction, showing motion artefacts. Void is black, fluid white, and rock/quartz grey. Centre: FBP ground-truth reconstruction, segmented to show void (white). Again, motion artefacts are clearly visible. Right: MAP-EM reconstruction, without assuming two-phase flow, using every tenth radiograph.

\section{RESULTS}

In order to test our MAP-EM algorithm, we require data from a "ground-truth" $\mu$ CT flow experiment. ${ }^{11}$ The goal of this experiment is to obtain data that can be reconstructed in two different ways: (i) with conventional $\mu \mathrm{CT}$ algorithms to obtain a reasonably-accurate "ground-truth"; and (ii) with our MAP-EM algorithm, to test its performance. The MAP-EM reconstruction will only be performed using a fraction of the recorded data, to simulate the more rapid nature of a standard $\mu \mathrm{CT}$ flow experiment.

A full description of the experimental process can be found in Ref. 11. In summary, a cylindrical Bentheimer plug $5 \mathrm{~mm}$ in diameter was fully saturated with fluid, and then drained at a rate of $0.025 \mu \mathrm{L} / \mathrm{min}$. During the 15 hour drainage process 133,920 radiographs were collected, each $512^{2}$ pixels with an exposure time of $0.4 \mathrm{~s}$, and an angular separation of 0.5 degrees. The source and detector were arranged such that the cone-angle was approximately 52 degrees; geometric magnification resulted in a voxel size of $12.8 \mu \mathrm{m}$.

The two-phase flow data was reconstructed three times: (i) from the full data set, using conventional FBP reconstruction; (ii) from only every tenth radiograph, using the empirically-derived dynamic tomography algorithm from Ref. 7; and (iii) from only every tenth radiograph, using the MAP-EM algorithm in Sec. 3.5. Figure 1 shows the reconstructed air distribution during drainage, verifying the results of the MAP-EM algorithm, and demonstrating that it yields the same improvements in temporal resolution as the empirically-derived algorithm in Ref. 7. The MAP-EM algorithm better reconstructed fine features than the empirically derived algorithm (see bottom middle of image). Furthermore, there are voxels in the FBP reconstruction that are partially filled with impermeable rock. These voxels are outside the support $\Omega$; the empirically-derived algorithm reconstructed them as fully-filled, whilst the MAP-EM algorithm correctly reconstructed them as only partially-filled with void. We note that as in Ref. 11, the data contained significant beam hardening. This made it difficult to accurately 
determine the support of the rock scaffold.

We intend to apply the MAP-EM algorithm to more complex systems, involving more than two fluid phases. Consequently, we wish to study the performance of the algorithm in the limiting case where the materials dictionary consists solely of "impermeable rock scaffold", and "other". To do so, we set $\alpha_{3}$ to zero in Eqn. (4). Figure 2 shows 2D slices through the resulting reconstructions, indicating that the MAP-EM algorithm may be useful in the study of Haines-jump dominated multiphase systems. Note that the motion artefacts present in the FBP reconstructions (top left, and top middle, Fig. 2) are not present in the MAP-EM reconstruction. As before, the MAP-EM algorithm uses only every tenth radiograph.

\section{CONCLUSION}

We have presented an expectation maximisation algorithm for the maximum a posteriori estimation of timeevolving multiphase fluid distributions in microporous materials (e.g. water drainage in Bentheimer sandstone). The algorithm uses experimental data collected at an X-ray $\mu \mathrm{CT}$ facility, and a priori information about the physics constraining the behaviour of the sample.

We have verified that when applied to the study of two-phase flow, this MAP-EM algorithm improves temporal resolution by a factor of ten compared to standard $\mu \mathrm{CT}$ reconstruction methods, with no corresponding loss of spatial resolution. Our previous approach to dynamic tomography was unable to account for "mixels": voxels which contain a mixture of fluid, void and impermeable rock. Experimental reconstructions demonstrate that the MAP-EM algorithm can correctly detect and reconstruct these mixels, and that the algorithm can be generalised to more complex physical systems such as multiphase flow.

\section{ACKNOWLEDGMENTS}

This research was supported under the Australian Research Council's Discovery Projects funding scheme (project number DP110102964). Thomas Li acknowledges receipt of an ANU University Research Scholarship. A/Prof.

Adrian Sheppard is the recipient of an Australian Research Council Future Fellowship (project number FT100100470).

\section{REFERENCES}

[1] Natterer, F., [The Mathematics of Computerized Tomography], Society for Industrial and Applied Mathematics, Philadelphia (2001).

[2] Caubit, C., Hamon, G., Sheppard, A. P., and Øren, P. E., "Evaluation of the reliability of prediction of petrophysical data through imagery and pore network modelling," in [22nd International Symposium of the Society of Core Analysts], Society of Core Analysts (October 2008). SCA2008-33.

[3] Kak, A. C. and Slaney, M., [Principles of Computerized Tomographic Imaging], SIAM, Philadelphia (2001).

[4] Lange, K. and Fessler, J. A., "Globally convergent algorithms for maximum a posteriori transmission tomography.," IEEE transactions on image processing : a publication of the IEEE Signal Processing Society 4(10), 1430-1438 (1995).

[5] Chen, G., Tang, J., and Leng, S., "Prior image constrained compressed sensing (piccs): A method to accurately reconstruct dynamic ct images from highly undersampled projection data sets," Med. Phys. Lett. 35, 660-663 (2008).

[6] Bonnet, S., Koenig, A., Roux, S., Hugonnard, P., Guillemard, R., and Grangeat, P., "Dynamic x-ray computed tomography," Proceedings of the IEEE 91, 1574-1587 (2001).

[7] Myers, G., Kingston, A., Varslot, T., Turner, M., and Sheppard, A., "Dynamic tomography with a priori information," Applied Optics 50, 3685-3690 (2011).

[8] OHalloran, R. L., Wen, Z., Holmes, J. H., and Fain, S. B., "Iterative projection reconstruction of timeresolved images using highly-constrained back-projection (hypr)," Magnetic Resonance in Medicine 59, 132-139 (2008).

[9] Mistretta, C. A., "Sub-nyquist acquisition and constrained reconstruction in time resolved angiography," Med. Phys. 38, 2975-2985 (2011). 
[10] Myers, G., Kingston, A., Varslot, T., Turner, M., and Sheppard, A., "Dynamic x-ray micro-tomography for real time imaging of drainage and imbibition processes at the pore scale," Proc. International Symposium of the Society of Core Analysts 2011 2011, SCA2011-27 (2011).

[11] Myers, G., Varslot, T., Kingston, A., Herring, A., and Sheppard, A., "Ground-truth verification of dynamic x-ray micro-tomography images of fluid displacement," Proc. SPIE, Developments in X-ray Tomography VIII 8506, 85060P (2012).

[12] Varslot, T., Kingston, A., Myers, G., and Sheppard, A., "High-resolution helical cone-beam micro-ct with theoretically-exact reconstruction from experimental data," Medical Physics 38, 5459 (2011).

[13] Kingston, A., Sakellariou, A., Varslot, T., Myers, G. R., and Sheppard, A., "Reliable automatic alignment of tomo- graphic projection data by passive auto-focus," Medical Physics 38, 4934 (2011).

[14] Myers, G., Kingston, A., Varslot, T., and Sheppard, A., "Extending reference scan drift correction to high-magnification high-cone-angle tomography," Optics Letters 36, 4809-4811 (2011).

[15] Sheppard, A., Latham, S., Middleton, J., Kingston, A., Myers, G., Varslot, T., Fogden, A., Sawkins, T., Cruikshank, R., Saadatfar, M., Francois, N., Arns, C., and Senden, T., "Techniques in helical scanning, dynamic imaging and image segmentation for improved quantitative analysis with x-ray micro-ct," Nuclear Instruments and Methods B 324, 49-56 (2014).

[16] Berg, S., Ott, H., Klapp, S. A., Schwing, A., Neiteler, R., Brussee, N., Makurat, A., Leu, L., Enzmann, F., Schwarz, J. O., Kersten, M., Irvine, S., and Stampanoni, M., "Real-time 3d imaging of haines jumps in porous media flow," Proceedings of the National Academy of Sciences 110, 3755-3759 (2013).

[17] DiCarlo, D. A., Cidoncha, J. I. G., and Hickey, C., "Acoustic measurements of pore-scale displacements.," Geophysical research letters 30, 1901 (2003).

[18] Haines, W. B., "Studies in the physical properties of soil. v. the hysteresis effect in capillary properties, and the modes of moisture distribution associated therewith," The Journal of Agricultural Science 20, 97-116 (1930).

[19] Larson, R. G., Davis, H. T., and Scriven, L. E., "Displacement of residual nonwetting fluid from porous media," Chemical Engineering Science 36, 75-85 (1981).

[20] Mohanty, K. K., Davis, H. T., and Scriven, L. E., "Physics of oil entrapment in water-wet rock," SPE Reservoir Engineering 2, 113-128 (1987).

[21] Daubechies, I., Defrise, M., and Mol, C. D., "An iterative thresholding algorithm for linear inverse problems with a sparsity constraint," Communications on Pure and Applied Mathematics LVII, 1413-1457 (2004). 